\title{
Self-Tuned Kernel Spectral Clustering for Large Scale Networks
}

\author{
Raghvendra Mall \\ Department of Electrical Engineering \\ ESAT-SCD \\ Katholieke Universiteit Leuven \\ Kasteelpark Arenberg 10 \\ B-3001 Leuven, Belgium \\ Email:rmall@esat.kuleuven.be
}

\author{
Rocco Langone \\ Department of Electrical Engineering \\ ESAT-SCD \\ Katholieke Universiteit Leuven \\ Kasteelpark Arenberg 10 \\ B-3001 Leuven, Belgium \\ rocco.langone@esat.kuleuven.be
}

\author{
Johan A.K. Suykens \\ Department of Electrical Engineering \\ ESAT-SCD \\ Katholieke Universiteit Leuven \\ Kasteelpark Arenberg 10 \\ B-3001 Leuven, Belgium \\ johan.suykens@esat.kuleuven.be
}

\begin{abstract}
We propose a parameter-free kernel spectral clustering model for large scale complex networks. The kernel spectral clustering (KSC) method works by creating a model on a subgraph of the complex network. The model requires a kernel function which can have parameters and the number of communities $k$ has be detected in the large scale network. We exploit the structure of the projections in the eigenspace to automatically identify the number of clusters. We use the concept of entropy and balanced clusters for this purpose. We show the effectiveness of the proposed approach by comparing the cluster memberships w.r.t. several large scale community detection techniques like Louvain, Infomap and Bigclam methods. We conducted experiments on several synthetic networks of varying size and mixing parameter along with large scale real world experiments to show the efficiency of the proposed approach.
\end{abstract}

Keywords-kernel spectral clustering, number of clusters, parameter-free spectral clustering

\section{INTRODUCTION}

In the modern era complex networks are ubiquitous. Their presence ranges from domains like road graphs, social networks, collaboration networks, e-mail networks, trust networks, biological networks to financial networks. Complex networks can be represented as a graph $G(V, E)$, where the vertices $V$ represent the nodes of the graph and the edges are represented by $E$. The nodes represent the entities in the network and the edges determine the relationship between these entities. Real world networks exhibit community like structure. This means that the nodes which are part of one community tend to be more densely connected within the community and sparsely connected between the communities. The problem of community detection has received a lot of attention [1], [2], [3], [4], [5], [6], [7], [8], [9], [10], [11], [12] and has often been framed as graph partitioning and graph clustering [13]. In this paper we refer to clusters and communities interchangeably.

Spectral clustering methods [9], [10], [11], [12] are widely used for community detection. Spectral clustering methods work by performing an eigen-decomposition of a normalized Laplacian matrix derived from the affinity matrix of the nodes in the network. The disadvantage of spectral clustering methods is that they require to compute and store the affinity matrix. As the size of network increases it becomes in-feasible to store the $N \times N$ affinity matrix where $N$ is the number of nodes. Also, these methods do not have an out-of-sample extension property. Hence, they cannot scale for very large networks (millions of nodes).

Recently, a kernel spectral clustering formulation based on weighted kernel principal component analysis (PCA) with a primal-dual framework was proposed in [14]. The method was extended for community detection in [7] and [22]. The KSC model requires a kernel function and the number of clusters $k$ has to be detected. For model selection, the proposed approach in [7] and [22] uses the concept of Modularity [16]. The Modularity criteria needs to store a $N \times N$ matrix which makes it in-feasible for large scale networks. An approach to apply KSC for big data networks was proposed in [15]. We use the concepts proposed in [15] to build the model. In this paper we propose to make the kernel spectral clustering approach parameter-free or in other words

- Use the normalized linear kernel function.

- Automatically detect the number of communities $(k)$ in the large scale network.

In section [I] a description of the KSC method for large scale networks is given. In section III] we explain the proposed approach for making KSC parameter-free. In section IV, we perform experiments on various synthetic and real world datasets. Section $\mathrm{V}$ provides conclusion to the paper.

\section{Kernel Spectral Clustering for Large Scale NETWORKS}

The KSC [14] and [7] method consists of three steps. The first step is building the model on the training data. The second step is to estimate the model parameters using validation data. The final step is the out-of-sample extension or community inference for unseen test nodes.

For building the model, the KSC method selects a subgraph which captures the inherent community structure of the network. Several approaches have been proposed for sampling a graph including [17], [18], [19], [20]. The Fast and Unique Representative Subset (FURS) selection approach 
was proposed in [18] and used in [15]. We use the FURS technique for training and validation set selection.

For large scale networks, the training data comprises the adjacency list of all the nodes $v_{i}, i=1, \ldots, N_{t r}$. Let the training set of nodes be represented by $V_{t r}$ and the training set cardinality be $N_{t r}$. The validation and test set of nodes are represented by $V_{\text {valid }}$ and $V_{\text {test }}$ respectively. The cardinality of these sets are $N_{\text {valid }}$ and $N_{\text {test }}$ respectively. These sets of adjacency lists can efficiently be stored in the memory as real world networks are highly sparse and there are limited connections for each node $v_{i} \in V_{t r}$. The maximum length of the adjacency list can be equal to $N$. This is the case when a node is connected to all the other nodes in a network.

\section{A. Model Building and overcoming any kernel parameters}

For $V_{t r}$ training nodes, $\mathcal{D}=\left\{x_{i}\right\}_{i=1}^{N_{t r}}$, such that $x_{i} \in \mathbb{R}^{N}$. Given $\mathcal{D}$ and a user-defined $k$ (maximum number of clusters in the network), the primal formulation of the weighted kernel PCA [14] is given by:

$$
\begin{array}{ll}
\min _{w^{(l)}, e^{(l)}, b_{l}} & \frac{1}{2} \sum_{l=1}^{k-1} w^{(l)^{\top}} w^{(l)}-\frac{1}{2 N_{t r}} \sum_{l=1}^{k-1} \gamma_{l} e^{(l)^{\top}} D_{\Omega}^{-1} e^{(l)} \\
\text { such that } & e^{(l)}=\Phi w^{(l)}+b_{l} 1_{N_{t r}}, l=1, \ldots, k-1
\end{array}
$$

where $e^{(l)}=\left[e_{1}^{(l)}, \ldots, e_{N_{t r}}^{(l)}\right]^{\top}$ are the projections onto the eigenspace, $l=1, \ldots, k-1$ indicates the number of score variables required to encode the $k$ communities, $D_{\Omega}^{-1} \in$ $\mathbb{R}^{N_{t r} \times N_{t r}}$ is the inverse of the degree matrix associated to the kernel matrix $\Omega . \Phi$ is the $N_{t r} \times d_{h}$ feature matrix, $\Phi=$ $\left[\phi\left(x_{1}\right)^{\top} ; \ldots ; \phi\left(x_{N_{t r}}\right)^{\top}\right]$ and $\gamma_{l} \in \mathbb{R}^{+}$are the regularization constants. We note that $N_{t r} \ll N$ i.e. the number of nodes in the training set is much less than the total number of nodes in the large scale network. The kernel matrix $\Omega$ is obtained by calculating the similarity between the adjacency list of each pair of nodes in the training set. Each element of $\Omega$, denoted as $\Omega_{i j}=K\left(x_{i}, x_{j}\right)=\phi\left(x_{i}\right)^{\top} \phi\left(x_{j}\right)$ is obtained by calculating the cosine similarity between the adjacency lists $x_{i}$ and $x_{j}$ which has been shown to be an effective similarity measure for large scale data [25]. Thus, $\Omega_{i j}=\frac{x_{i}^{\top} x_{j}}{\left\|x_{i}\right\|\left\|x_{j}\right\|}$ can be calculated efficiently using notions of set unions and intersections. This corresponds to using a normalized linear kernel function $K(x, z)=\frac{x^{\top} z}{\|x\|\|z\|}$ [24]. The clustering model is then represented by:

$$
e_{i}^{(l)}=w^{(l)^{\top}} \phi\left(x_{i}\right)+b_{l}, i=1, \ldots, N_{t r}
$$

where $\phi: \mathbb{R}^{N} \rightarrow \mathbb{R}^{d_{h}}$ is the mapping to a high-dimensional feature space $d_{h}, b_{l}$ are the bias terms, $l=1, \ldots, k-1$. However, for large scale networks we can utilize the explicit expression of the underlying feature map and $d_{h}=N$. Since the KSC formulation is valid for any positive definite kernel, we use a normalized linear kernel function to avoid any kernel parameter. The projections $e_{i}^{(l)}$ represent the latent variables of a set of $k-1$ binary cluster indicators given by $\operatorname{sign}\left(e_{i}^{(l)}\right)$ which can be combined with the final groups using an encoding/decoding scheme. The dual problem corresponding to this primal formulation is:

$$
D_{\Omega}^{-1} M_{D} \Omega \alpha^{(l)}=\lambda_{l} \alpha^{(l)}
$$

where $M_{D}=I_{N_{t r}}-\left(\frac{\left(1_{N_{t r}} 1_{N_{t r}}^{\top} D_{\Omega}^{-1}\right)}{1_{N_{t r}}^{\top} D_{\Omega}^{-1} 1_{N_{t r}}}\right)$. The $\alpha^{(l)}$ are the dual variables and the kernel function $K: \mathbb{R}^{N} \times \mathbb{R}^{N} \rightarrow \mathbb{R}$ plays the role of similarity function.

\section{B. Encoding/Decoding Scheme}

Ideally when the communities are non-overlapping, we will obtain $k$ well separated clusters and the normalized Laplacian has $k$ piece-wise constant eigenvectors. This is because the multiplicity of the largest eigenvalue i.e. 1 is $k$ as depicted in [23]. In case of KSC due to the centering matrix $M_{D}$ the eigenvectors have zero mean and the optimal threshold for binarizing the eigenvectors is self-determined (equal to $0)$. So we need $k$-1 eigenvectors. In the eigenspace every cluster $A_{p}, p=1, \ldots, k$ is a point and is represented with a unique codebook $c_{p} \in\{-1,1\}^{k-1}$. To obtain this codebook $\mathcal{C B}=\left\{c_{p}\right\}_{p=1}^{k}$ we transform the rows of the projected vector matrix obtained from the training data by binarizing it i.e. $\left[\operatorname{sign}\left(e^{(1)}\right), \ldots, \operatorname{sign}\left(e^{(k-1)}\right)\right]$. The codebook set $\mathcal{C B}$ is obtained by selecting the top $k$ most frequent codebook vectors. However, in real world networks the communities do overlap and we do not have piece-wise constant eigenvectors.

For the matrix $D^{-1} M_{D} \Omega$ each eigenvector contains information about binary clustering. However, using the one versus all encoding scheme, the number of score variables needed to encode $k$ clusters is $k-1$. The decoding scheme consists of comparing the cluster indicators obtained in the validation/test stage with the codebook and selecting the nearest codebook based on Hamming distance. This scheme corresponds to the ECOC decoding procedure [26] and is used in out-of-sample extensions as well. The out-of-sample extension is based on the score variables which correspond to the projections of the mapped out-of-sample points onto the eigenvectors found in the training stage. The cluster indicators can be obtained by binarizing the score variables for the out-of-sample points as:

$$
\operatorname{sign}\left(e_{\text {test }}^{(l)}\right)=\operatorname{sign}\left(\Omega_{\text {test }} \alpha^{(l)}+b_{l} 1_{N_{\text {test }}}\right)
$$

where $l=1, \ldots, k-1, \Omega_{\text {test }}$ is the $N_{\text {test }} \times N_{t r}$ kernel matrix evaluated using the test points with entries $\Omega_{r, i}=K\left(x_{r}, x_{i}\right)$, $r=1, \ldots, N_{\text {test }}$ and $i=1, \ldots, N_{t r}$. This natural extension to out-of-sample points is the main advantage of $\mathrm{KSC}$. In this way the clustering model can be trained, validated and tested in an unsupervised learning procedure. For the test set we use the entire network. If the network cannot fit in memory, we divide it into chunks to calculate the cluster memberships for each test chunk in parallel.

\section{Model Selection or Estimation of $k$}

In [15], the authors proposed a criterion called Balanced Angular Fit $(B A F)$ which tries to estimate the number of clusters using the validation set of nodes. The $B A F$ criterion is based on the principle that the nodes which belong to the same cluster have nearly zero or very small angular distance in the eigenspace w.r.t. each other in comparison to the nodes belonging to different clusters. To determine the number of clusters, the end-user provides a maximum possible number of clusters (maxk). The KSC model is built using this maxk i.e. 
we perform an eigen-decomposition of the $D^{-1} M_{D} \Omega$ matrix and select the top maxk eigenvectors. The $B A F$ criterion is iteratively evaluated for $k=2$ to $k=\max k-1$. The value of $k$ for which the $B A F$ is maximum is provided to the end-user as the ideal number of clusters. The method works well but requires to iterate over all values of $k$ from 2 to $\max k-1$. Hence, it doesn't automatically determine the ideal value of $k$ in a few steps. Another approach to automatically determine the ideal value of $k$ was proposed in [11] using the structure of the eigenvectors. However, the underlying assumption is that the eigenvector matrix $Z$ comprises $k$ piece-wise constant eigenvectors and there is at most one non-zero entry in every row. Both these assumptions do not hold for the KSC model for real world overlapping networks. Algorithm 1 provides a summary of the Original KSC method.

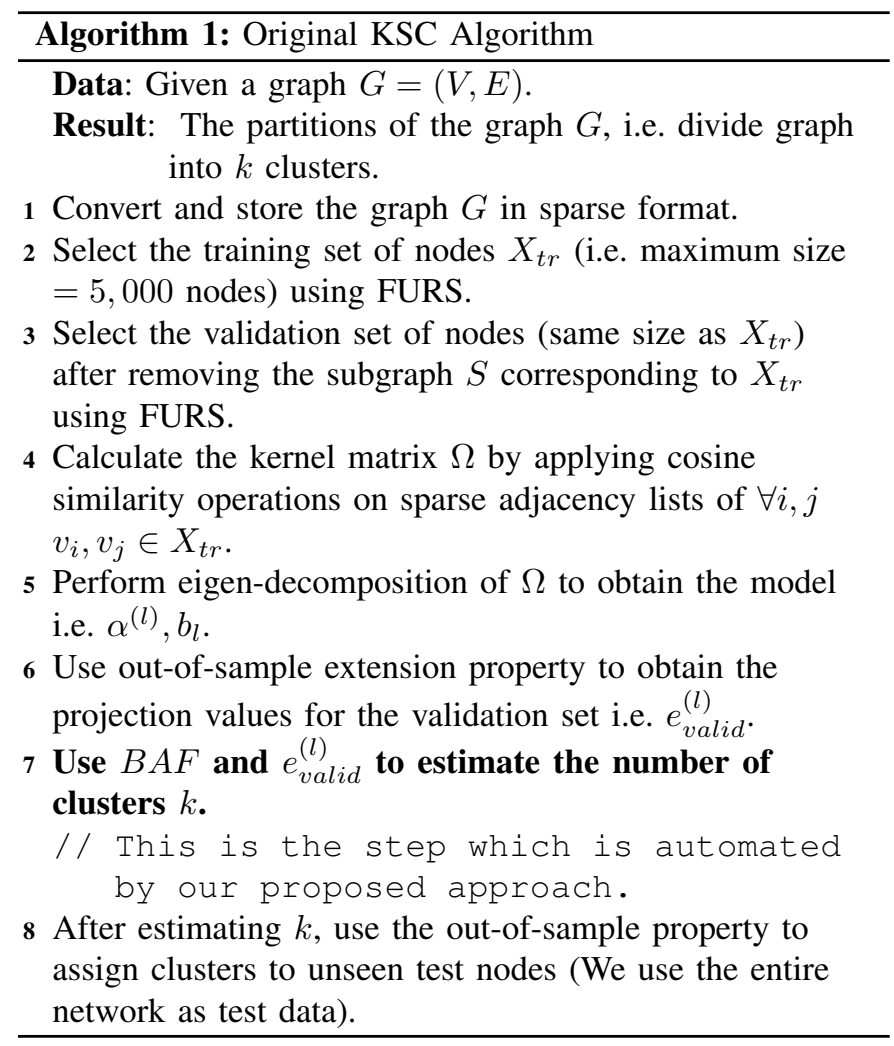

\section{Self-Tuned Kernel Spectral Clustering}

In section II-A we have shown that for large scale networks the normalized linear kernel function is sufficient. The only parameter left is the number of clusters to be identified $(k)$. We propose a novel technique to determine the number of clusters $(k)$ automatically in a few steps. We exploit the projections of the validation nodes in the eigenspace. The proposed approach is based on the simple idea that the projections of the validation nodes which belong to the same cluster have very small or nearly zero angular distance from each other in comparison with the nodes belonging to different clusters.

Before we explain the technique, we mention a few prerequisites. All the experiments were performed on a single machine with $8 \mathrm{~Gb}$ RAM, 2.4Ghz Intel I-7 processor. The maximum size matrix that can be stored in memory is $5,000 \times$ 5,000 . The training set size is fixed as $N_{t r}=\min (15 \%$ of $N, 5000)$ i.e. minimum between $15 \%$ of the total nodes in the network $(N)$ and 5,000 nodes. The subset size of $15 \%$ of the nodes in the network is chosen as per experimental analysis in [21]. We set the validation set size $N_{\text {valid }}$ to be the same as $N_{t r}$. We put the condition that minimum size of a cluster is MinCsize $=\max \left(0.01 \%\right.$ of $\left.N_{\text {valid }}, 5\right)$ i.e. maximum between $0.01 \%$ of the number of nodes in the validation set and 5 nodes. Since for estimation of the number of clusters we use the validation set, we put the minimum size constraint on the validation set. This constraint is to prevent the selection of outlier groups of small size as a cluster. Thus, suppose the training set size is 5,000 nodes then the minimum size of cluster has to be 5 . The maxk value is determined as $\left\lceil\frac{N_{t r}}{\text { MinCsize }}\right\rceil$. Thus, if the minimum size of the cluster is 5 and maximum size of the validation set is 5,000 then the maximum number of clusters that can be detected is 1,000 . The runtime complexity of KSC approach is $\mathrm{O}\left(N_{t r}^{3}\right)+\mathrm{O}\left(N_{t r} \times N_{t e s t}\right)$. However, testing can be done in parallel and the second term in time complexity can be reduced significantly.

\section{A. Identifying the number of clusters}

We obtain the projection of the validation nodes $v_{j} \in V_{\text {valid }}$ in the eigenspace using the out-of-sample extension and binarize the score variables as follows:

$$
\operatorname{sign}\left(e_{\text {valid }}^{(l)}\right)=\operatorname{sign}\left(\Omega_{\text {valid }} \alpha^{(l)}+b_{l} 1_{N_{\text {valid }}}\right)
$$

Let the latent variable matrix be represented as $E$. The size of the this matrix is $N_{\text {valid }} \times(\operatorname{maxk}-1)$. The matrix $E$ is defined as $\left[e_{1}, \ldots, e_{N_{\text {valid }}}\right]^{\top}$. We then create a new symmetric affinity matrix $A$ from the latent variable matrix $E$. Since we already stated that the maximum size of the validation set is 5,000 nodes, the maximum size of the $A$ matrix is $5,000 \times$ 5,000 . The affinity matrix is build using cosine distance as:

$$
A(i, j)=\operatorname{CosDist}\left(e_{i}, e_{j}\right)=1-\cos \left(e_{i}, e_{j}\right)=1-\frac{e_{i}^{\top} e_{j}}{\left\|e_{i}\right\|\left\|e_{j}\right\|}
$$

The $\operatorname{CosDist}()$ function can take values between $[0,2]$. We use the concept that nodes which belong to the same community have smaller or nearly zero angular distance w.r.t the nodes within the community and more angular distance w.r.t nodes belonging to different communities. Thus, the nodes which belong to the same cluster will have very small or nearly zero $\operatorname{CosDist}\left(e_{i}, e_{j}\right), \forall i, j$ in the same cluster.

Figure 1 highlights this idea on a synthetic network of 1,000 nodes generated by the software proposed in [2] with mixing parameter $\mu=0.1$. The mixing parameter implies the extent of overlap in the communities with smaller values representing less overlap. In Figure 1a, we obtain the projections of the validation nodes in 2-dimensional space as we set $\operatorname{maxk}=$ 3 for the purpose of visualization. Figure $1 b$ showcases the presence of a block diagonal structure for the affinity matrix $A$. To obtain this block diagonal matrix we used the groundtruth cluster memberships for the validation set of nodes. We then sort the validation nodes using the groundtruth information on 


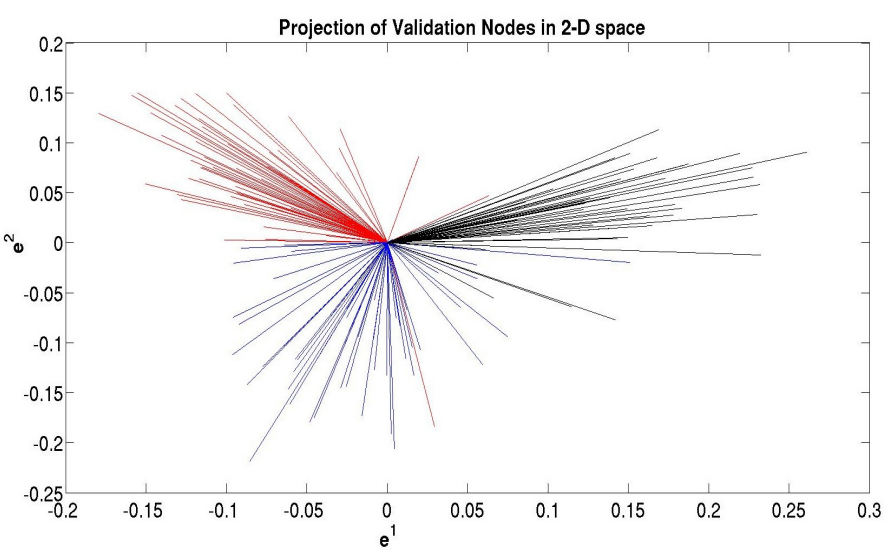

(a) Validation node projections

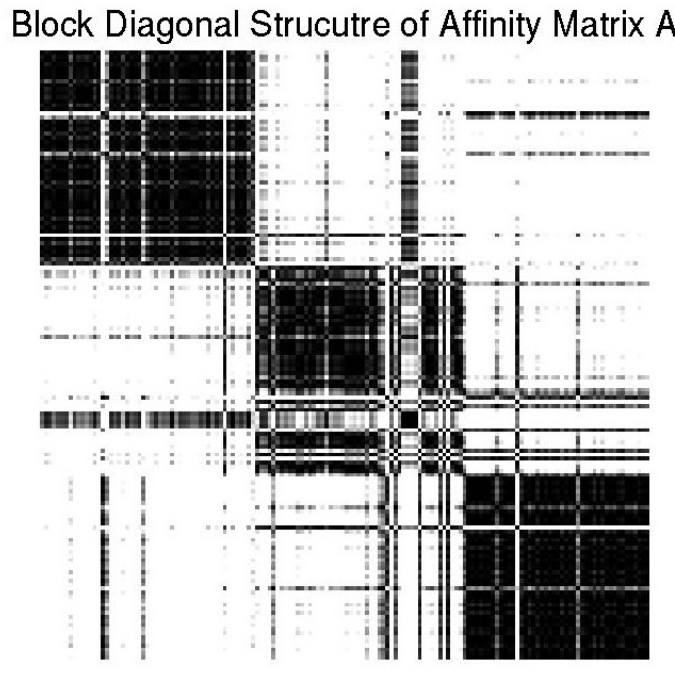

(b) Affinity matrix $A$ w.r.t. groundtruth

Fig. 1: First two steps for identifying the number of clusters in a given network

the basis of their cluster affiliation and construct the affinity matrix $A$. The black shaded regions represent the part of the affinity matrix where the $\operatorname{Cos} \operatorname{Dist}\left(e_{i}, e_{j}\right)$ values are zero and nearly equal to zero. The aim is to identify the number of such block diagonals which determine the number of clusters $k$ in the given network.

Real world networks have overlapping communities. However, the extent of overlap in these communities is not known beforehand. Also the groundtruth is not available in most real world large scale networks. Thus the affinity matrix $A$, though contains a block-diagonal structure, need not necessarily be ordered in block-diagonal format. We observe from Figure 1a that projection of nodes which belong to the same cluster do not necessarily have zero angular distance due to presence of overlap. But the angle between two projection vectors say $e_{i}$ and $e_{j}$ belonging to the $p^{t h}$ cluster $\left(C_{p}\right)$ is mostly less than $90^{\circ}$ leading to $0 \leq \cos \left(e_{i}, e_{j}\right) \leq 1$. After little algebraic manipulation we obtain that $0 \leq \operatorname{CosDist}\left(e_{i}, e_{j}\right) \leq 1$ for two nodes $i$ and $j$ belonging to cluster $p\left(C_{p}\right)$.

Since the extent of overlap in terms of angular distance is not known beforehand we setup a threshold $t d=$ $[0.1,0.2, \ldots, 1]$. We need to investigate only 10 different threshold values which is much less than iteratively solving for each $k=2$ to $k=\max k-1$ in increments of 1 . Generally as the value of threshold increases the number of clusters decreases as more validation nodes satisfy the threshold and fall in the same cluster. For a given value of threshold $t \in t d$, we first identify that validation node $\left(e_{i}\right)$ for which the number of nodes with $\operatorname{CosDist}\left(e_{i}, e_{j}\right) \leq t$ is maximum. We keep the count of this number of nodes in a vector. We obtain the indices of all those validation nodes and shrink the affinity matrix $A$ by removing the rows and columns corresponding to these indices. We repeat this process recursively till it results in an empty matrix. This procedure is equivalent to identifying the block diagonal structure. As a result of this procedure for each value of threshold $t$, we obtain a vector $\left(\right.$ Size $\left._{t}\right)$ containing information about the size of clusters for that value of threshold $t$.

In order to determine the ideal number of clusters $k$, we use the notion of entropy and balance w.r.t. the size of the clusters. For each value of threshold $t$ we have the vector Size $C_{t}$. We also put the constraint that the minimum size of a cluster is greater than or equal to MinCsize. So we remove from Size $_{t}$ vector all the size of clusters which are less than MinCsize. The Shannon entropy for a discrete set of probabilities is defined as:

$$
H(s)^{t}=-\sum_{i=1}^{k} p\left(s_{i}\right)^{t} \log \left(p\left(s_{i}^{t}\right)\right), \forall t \in t d
$$

where $H(s)^{t}$ is the entropy corresponding to the vector Size $C_{t}$ for the threshold $t$. Here $s_{i}^{t}$ is the size of the $i^{t h}$ cluster and $p\left(s_{i}^{t}\right)$ is defined as ratio between the size of $i^{t h}$ cluster corresponding to threshold $t$ and the size of the validation set $\left(N_{\text {valid }}\right)$. Entropy values are higher when there is a larger number of uniformly sized clusters.

Balance is generally defined as the ratio between the minimum size cluster in $S_{i z e} C_{t}$ and the maximum size cluster in $S_{i z e} C_{t}$. The aim of using balance is to prevent one or more large community from being superior over all other communities. Higher balance promotes uniformly sized clusters and prevents few extremely large communities from being dominant. This leads to more number of communities. In this paper, we use the overall balance criterion which is the sum of balance for all the clusters in $\operatorname{Size}_{t}$ and defined as:

$$
B(s)^{t}=\sum_{i=1}^{k} \frac{s_{i}^{t}}{\max \left(s_{1}^{t}, \ldots, s_{k}^{t}\right)}, \forall t \in t d
$$

where $s_{i}^{t}$ represents the size of the $i^{t h}$ cluster corresponding to threshold $t$. The denominator in (7) refers to maximum sized cluster for the threshold $t$. The higher the overall balance in the clusters, the closer the value of $B(s)^{t}$ is to $k$. 
We calculate the entropy and overall balance for each of these $S_{i z e} C_{t}$ and use the harmonic mean or an F-measure to determine the best of both entropy and overall balance as their values are comparable. The F-measure is defined as:

$$
F(s)^{t}=\frac{2 H(s)^{t} B(s)^{t}}{H(s)^{t}+B(s)^{t}}, \forall t \in t d
$$

where $H(s)^{t}$ and $B(s)^{t}$ represents the entropy and expected balance for threshold $t$. We evaluate $F(s)^{t}$ for all values of threshold $t \in t d$ and output that value of threshold $t$ say maxt for which $F(s)^{t}$ is maximum. The number of clusters $(k)$ is obtained from the vector $S i z e C_{\operatorname{maxt}}$ and is equal to the number of terms in this vector. Algorithm 2 summarizes the proposed method for identifying the number of clusters $(k)$ in a given large scale network.

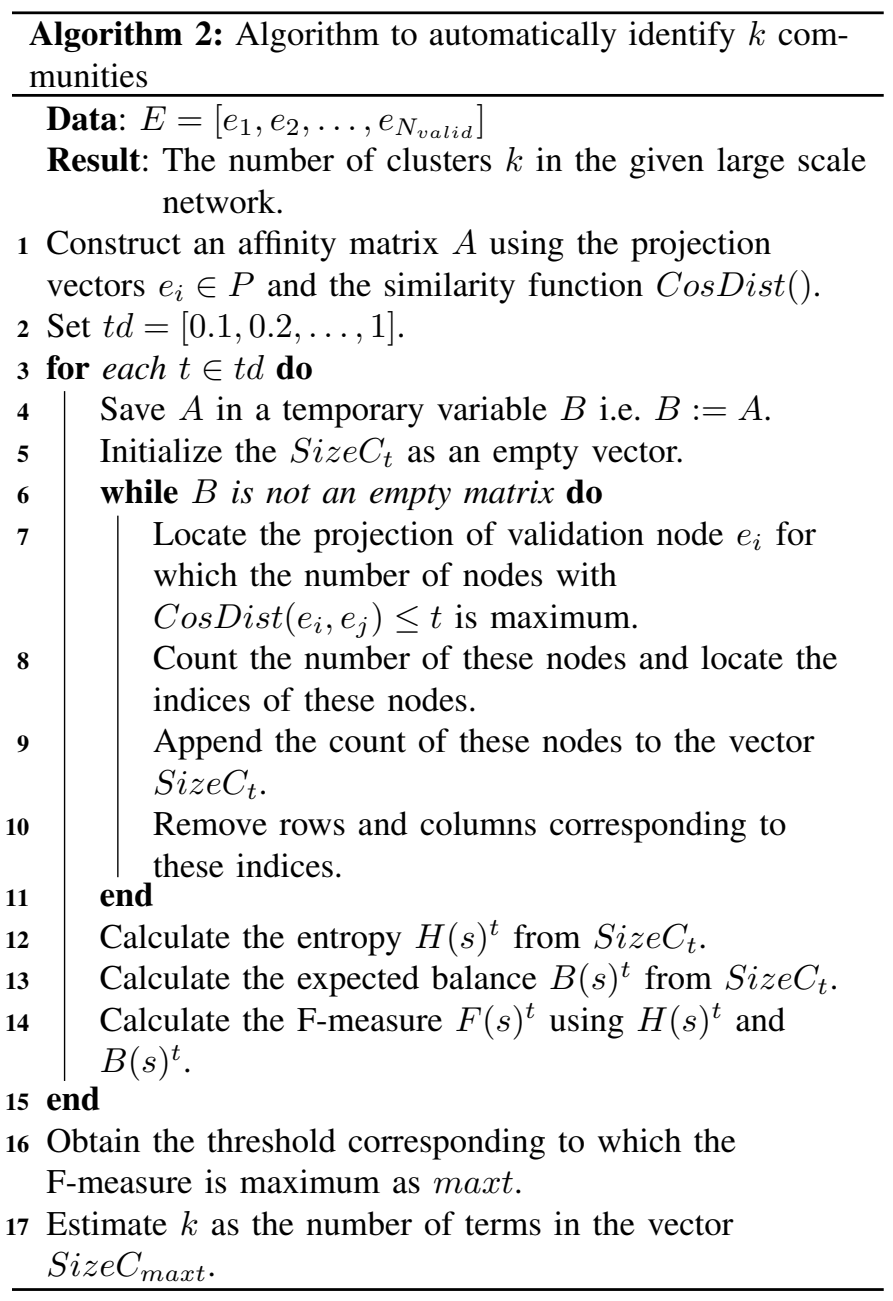

\section{EXPERIMENTS}

We conducted experiments on several synthetic and real world networks. The synthetic networks are generated from the software proposed in [2] with varying the size of the network and the mixing parameter $\mu$. The mixing parameter contains information about the extent of overlap between the communities in the large scale network.

\section{A. Experiments on Synthetic networks}

Figures 23, 4 and 5 show the result of our proposed approach for identifying the number of clusters $k$ on synthetic networks made of 5,000, 10,000, 25, 000 and 50,000 nodes respectively. We show the results for two different mixing parameters $\mu=0.1$ and $\mu=0.5$. For smaller $\mu$ the extent of overlap is less in comparison to higher value of $\mu$ and detecting communities is less difficult.

From Figures 2a, 2b, 3a, 3b, 4a, 4b, 5a and 5b, we show that the proposed method identifies exactly or close to the exact number of clusters present in the network even when the extent of overlap is high. The adjusted rand index (ARI) [27] values are also quite high suggesting that the clusters obtained via KSC are meaningful. We also observe that the threshold corresponding to which we obtain the maximum F-measure is related to the mixing parameter $\mu$. For the smaller mixing parameter $(0.1)$ the threshold value $t$ for which the F-measure is maximum is less than or equal to the threshold value corresponding to higher mixing parameter 0.5 as observed from Figures $2 \mathrm{c}, 2 \mathrm{~d}, 3 \mathrm{c}, 3 \mathrm{~d}, 4 \mathrm{c}, 4 \mathrm{~d}, 5 \mathrm{c}$ and $5 \mathrm{~d}$.

\section{B. Experiments on Real World Datasets}

We conducted experiments on several real-world large scale networks ranging from trust networks (PGPnet), collaboration networks (Cond-mat), citation networks (HepPh), communication networks (Enron), social networks (Epinion), web graphs (Youtube), actor networks (Imdb-actor) to road networks (roadCA). Details about these networks are available at http://snap.stanford.edu/data/index.html. Table I provides a few key statistics of each dataset.

\begin{tabular}{|c|c|c|c|}
\hline Dataset & Vertices & Edges & CCF \\
\hline PGPnet & 10,876 & 39,994 & 0.008 \\
Cond-mat & 23,133 & 186,936 & 0.6334 \\
HepPh & 34,401 & 421,578 & 0.1457 \\
Enron & 36,692 & 367,662 & 0.497 \\
Epinions & 75,879 & 508,837 & 0.2283 \\
Imdb-actor & 383,640 & $1,342,595$ & 0.453 \\
Youtube & $1,134,890$ & $2,987,624$ & 0.173 \\
roadCA & $1,965,206$ & $5,533,214$ & 0.0464 \\
\hline
\end{tabular}

TABLE I: Vertices (V), Edges (E) and Clustering Coefficients $(\mathrm{CCF})$ for each dataset

We use the entire large scale network as test set in our experiments. We use the clusters obtained by Louvain [8], Infomap [6] and Bigclam [28] methods as groundtruth for these networks. We compare the clusters obtained via the Original KSC method w.r.t. these groundtruth clusters. Then we modify the Original KSC method by automatically determining the number of clusters $(k)$. We combine our proposed approach with the original out-of-sample extension property of the KSC model to obtain the Self-Tuned KSC (ST-KSC) method.

In order to perform a comprehensive evaluation, once we automatically obtain the value of $k$, we perform $k$-means on the projections in the eigenspace for the validation set to obtain the centroids. We obtain the projections of the test set in the eigenspace and assign the cluster membership corresponding to the centroid to which it is the closest. This method is referred as Self-Tuned $k$-means KSC (ST $k$-means KSC). We 


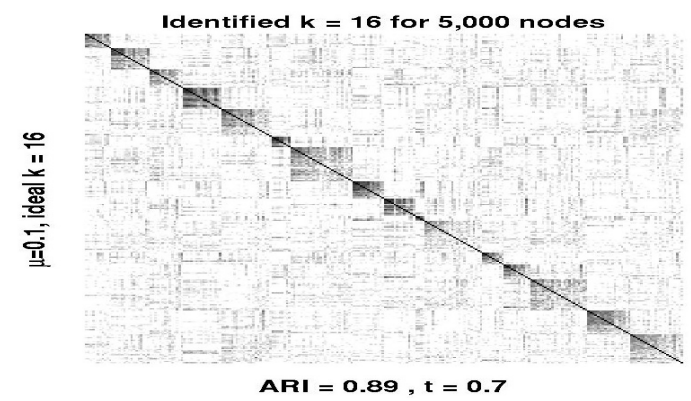

(a) $A$ w.r.t. groundtruth

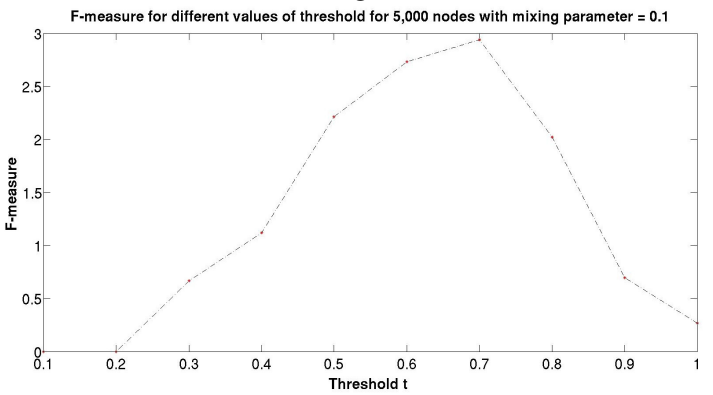

(c) F-measure vs Threshold

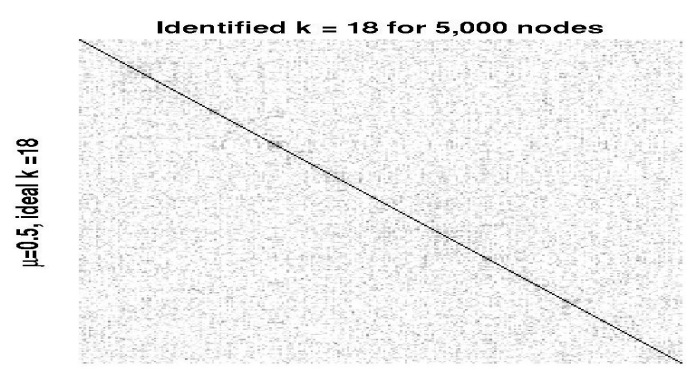

ARI $=0.19, t=0.8$

(b) $A$ w.r.t. groundtruth

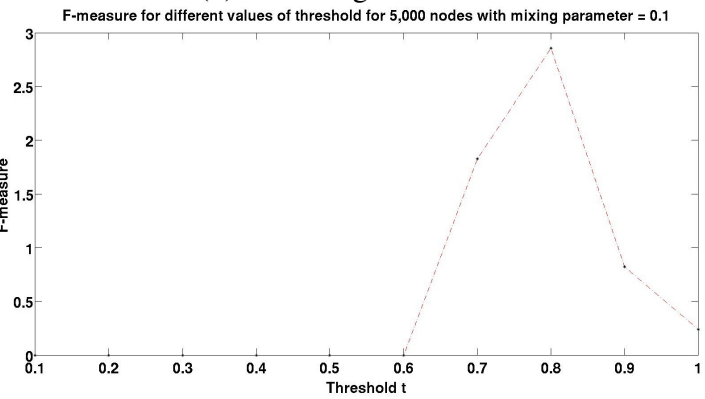

(d) F-measure vs Threshold

Fig. 2: Self-Tuned KSC on a synthetic network of 5,000 nodes for mixing parameter values 0.1 and 0.5

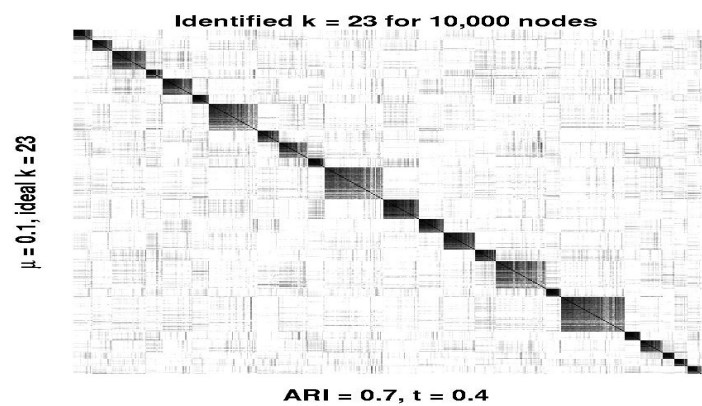

(a) $A$ w.r.t. groundtruth

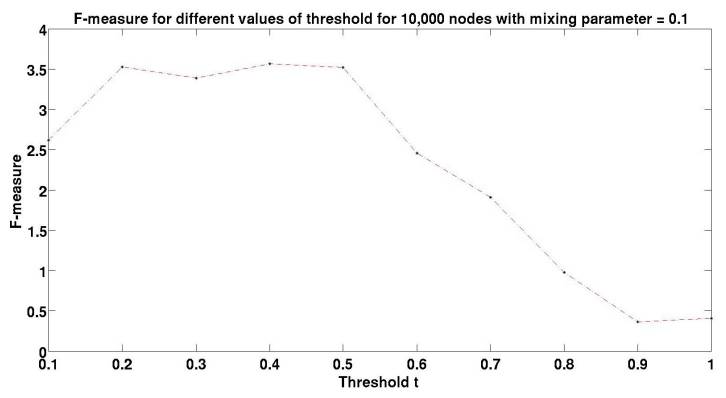

(c) F-measure vs Threshold

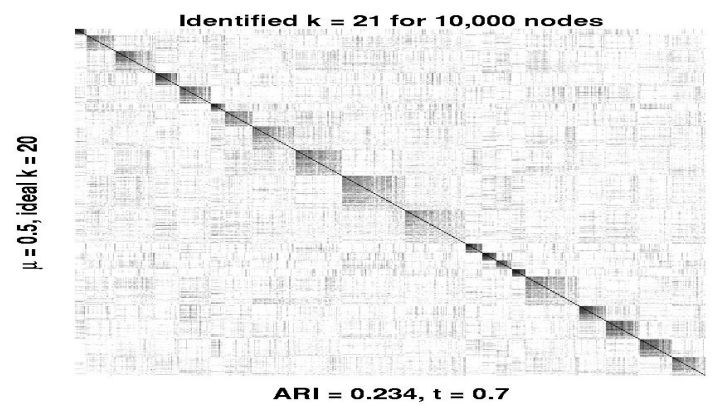

(b) $A$ w.r.t. groundtruth

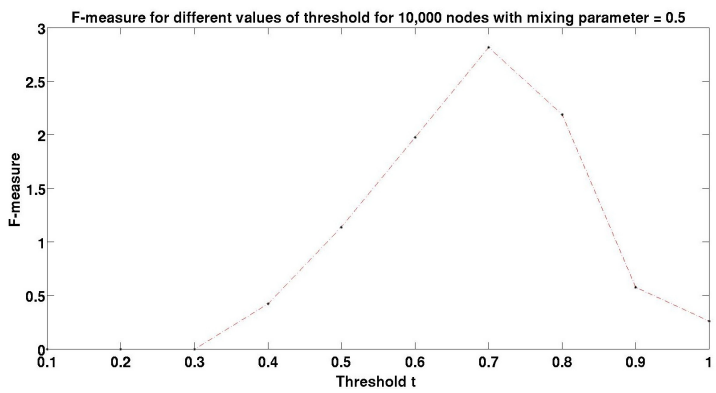

(d) F-measure vs Threshold

Fig. 3: Self-Tuned KSC on a synthetic network of 10,000 nodes for mixing parameter values 0.1 and 0.5

then compare the cluster memberships obtained via ST-KSC and ST $k$-means KSC w.r.t. the community affiliations via Louvain, Infomap and Bigclam method respectively.

For evaluating the cluster memberships we use three information theoretic measures: mutual information (MI), variation of information (VI) and adjusted rand index (ARI). These are standard cluster quality evaluation metrics given two list of cluster memberships and are described in detail in [29].
Table II evaluates the clusters obtained via the original KSC method, the proposed ST-KSC method and ST $k$-means KSC methods w.r.t. the clusters obtained by Louvain, Infomap and Bigclam methods. Since the partition obtained by methods like Louvain and Infomap method are not unique, we perform 10 randomizations and report the mean VI, ARI and MI values. Figure $6 \mathrm{a}$ and $6 \mathrm{~b}$ shows the variations for these evaluation metrics in case of Enron and roadCA networks respectively. 


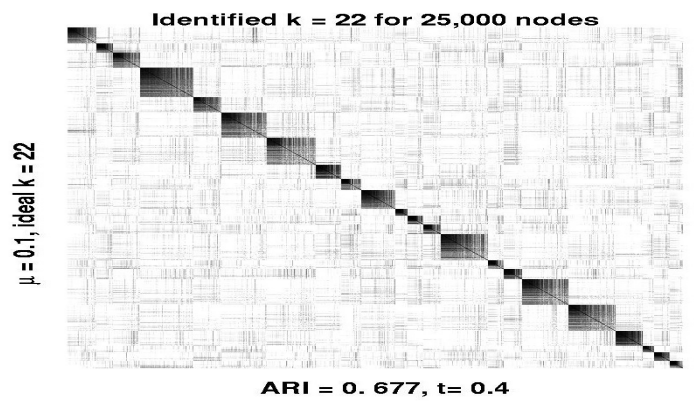

(a) $A$ w.r.t. groundtruth

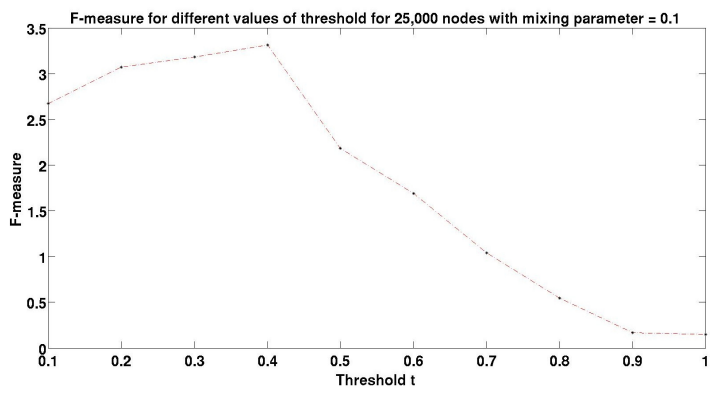

(c) F-measure vs Threshold

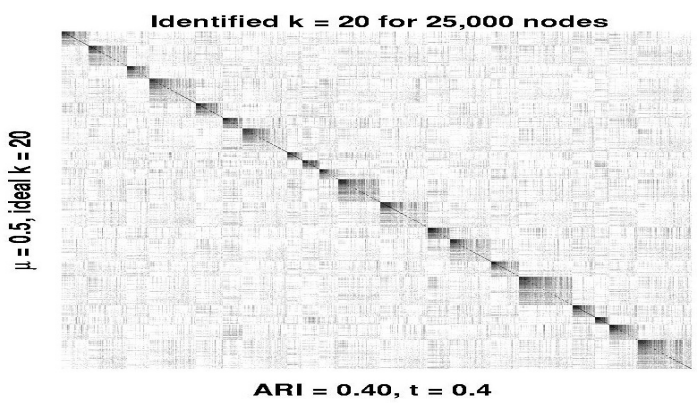

(b) $A$ w.r.t. groundtruth

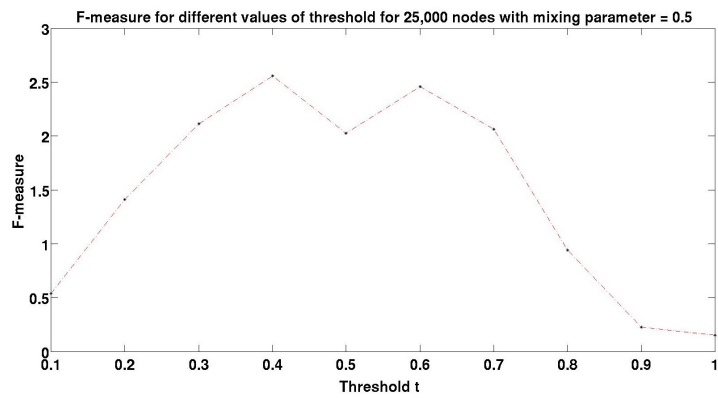

(d) F-measure vs Threshold

Fig. 4: Self-Tuned KSC on a synthetic network of 25,000 nodes for mixing parameter values 0.1 and 0.5

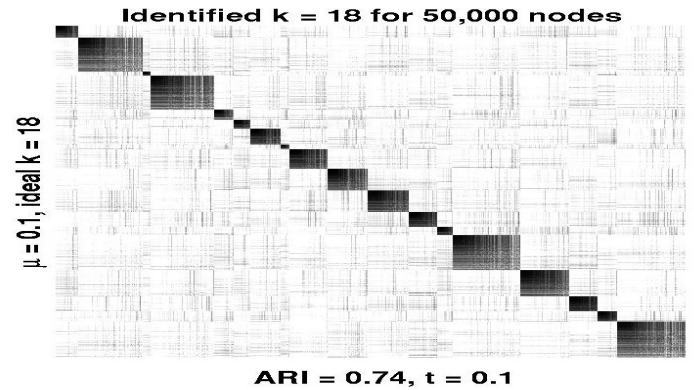

(a) $A$ w.r.t. groundtruth

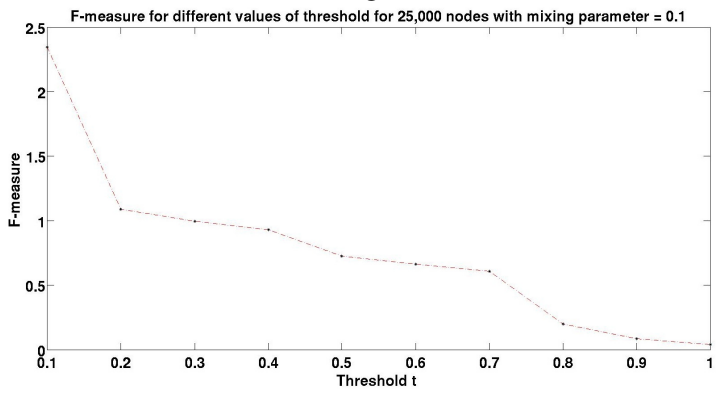

(c) F-measure vs Threshold

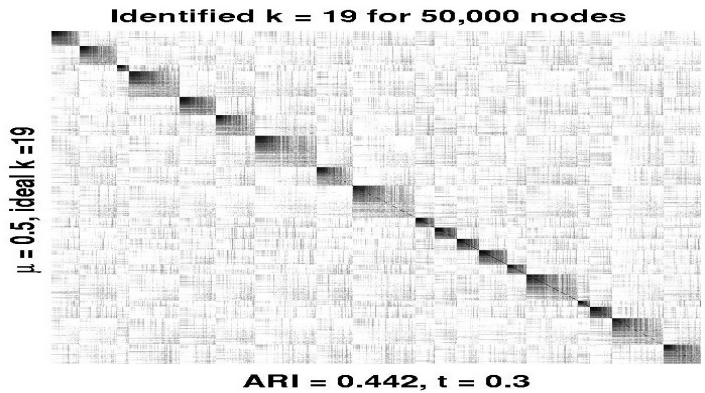

(b) $A$ w.r.t. groundtruth

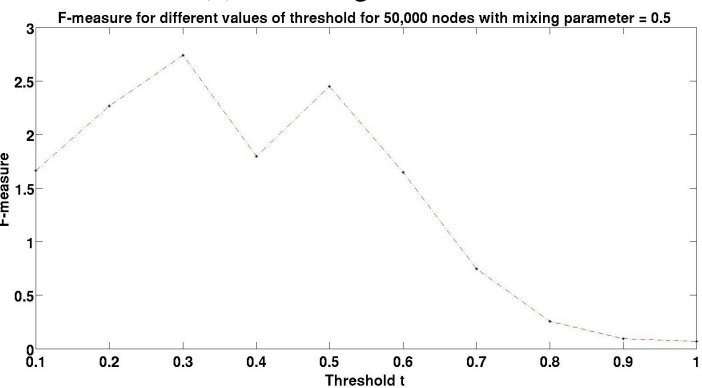

(d) F-measure vs Threshold

Fig. 5: Self-Tuned KSC on a synthetic network of 50,000 nodes for mixing parameter values 0.1 and 0.5

From Table II we observe that the absolute values of ARI are quite small for most networks as it is heavily dependent on the number of clusters. For methods like Louvain and Infomap method, the number of clusters found are much more than that for KSC methods which lead to smaller ARI. From Table II we observe that clusters obtained by the Original KSC method generally have better variation of information (VI) corresponding to the clusters obtained via Louvain, Infomap and Bigclam methods. This is because the Original KSC method using the $B A F$ evaluation metric is biased to produce small number of clusters as observed from Table II However, due to this bias it results in poor ARI and MI values for most of the real world large scale networks. The clusters obtained by the two self-tuned methods have better ARI and MI values. The ST-KSC method results in better quality clusters for PGPnet, Enron, Imdb-actor and roadCA networks when the 
Comparison of various metrics between ST-KSC and Louvain, ST-KSC and Infomap, ST-KSC and Bigclam method
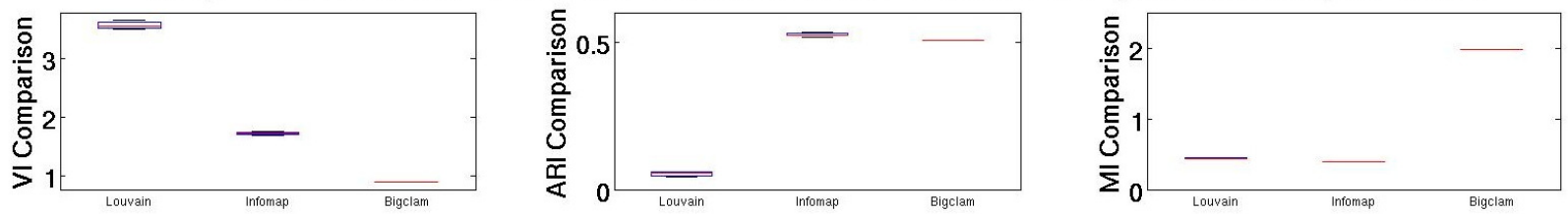

Comparison of various metrics between Original KSC and Louvain, Original KSC and Infomap, Original KSC and Bigclam method
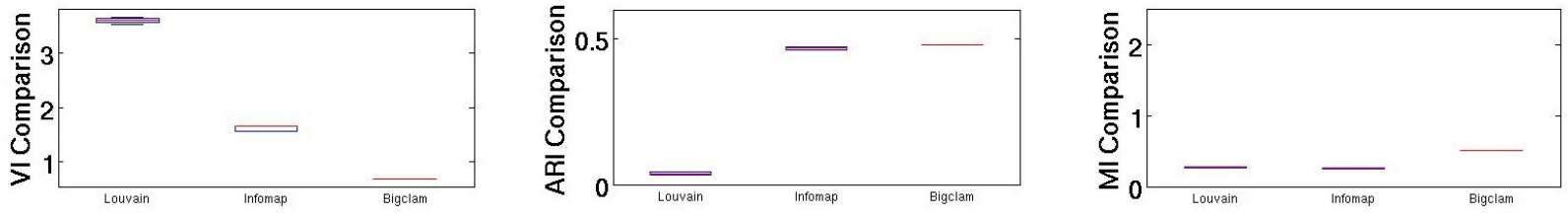

Comparison of various metrics between ST k-means KSC and Louvain, ST k-means KSC and Infomap, ST k-means KSC and Bigclam method
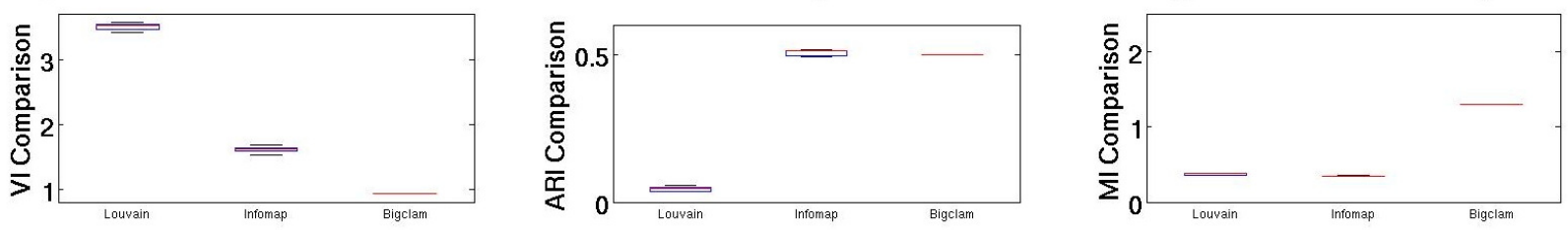

(a) For Enron network

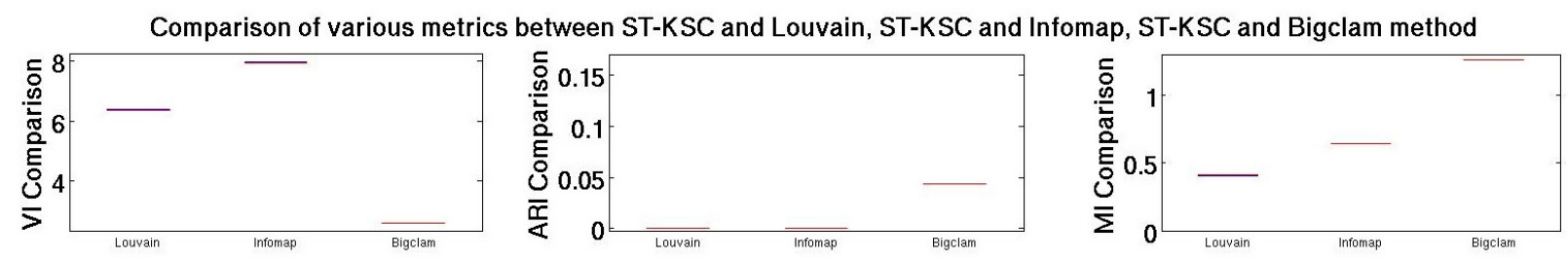

Comparison of various metrics between Original-KSC and Louvain, Original-KSC and Infomap, Original-KSC and Bigclam method
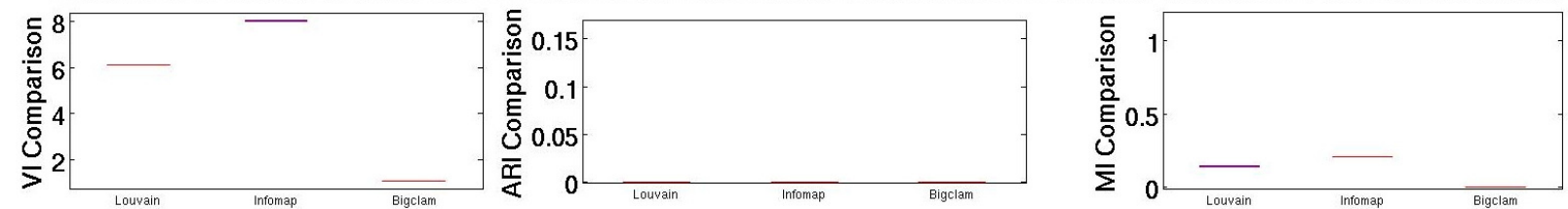

Comparison of various metrics between ST k-means KSC and Louvain, ST k-means KSC and Infomap, ST k-means KSC and Bigclam method
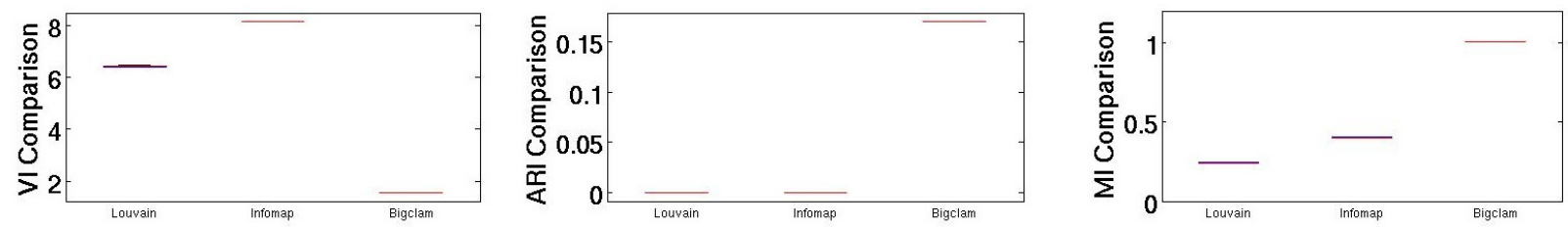

(b) For roadCA network

Fig. 6: Variation of evaluation metrics (VI, ARI and MI) for various methods for Enron 6a and roadCA 6b network. It shows that ST-KSC method gives best results for Enron network and ST $k$-means KSC method provides best results for roadCA network.

clusters are evaluated w.r.t. the clusters obtained by Louvain, Infomap and Bigclam methods. The evaluation metrics for which ST-KSC method performs better is ARI and MI. The ST $k$-means KSC method results in better quality clusters for Cond-mat, HepPh and Youtube networks w.r.t to the quality metrics ARI and MI.

Figure 6 showcases the results of the various quality metrics for the Enron and roadCA networks. Figure 6a shows that the clusters obtained by all the KSC methods are dissimilar from those obtained by Louvain method because of the high VI, low ARI and low MI values. However, the clusters obtained by the KSC methods are more similar to the ones obtained by Infomap and Bigclam methods. We observe this from 6a due to the low VI, high ARI and better MI values. In case of the Bigclam method, the clusters obtained are highly similar to that obtained by all the KSC methods. The clusters produced by the ST-KSC method in particular with VI (0.91), ARI (0.51) and MI (1.97) is the most similar to the clusters produced by 


\begin{tabular}{|c|c|c|c|c|c|c|c|c|c|c|c|c|}
\hline & & \multicolumn{3}{|c|}{ Original KSC } & \multicolumn{4}{|c|}{ ST-KSC } & \multicolumn{4}{|c|}{ ST $k$-means KSC } \\
\hline & & \begin{tabular}{|l|l|}
$k$ & $\mathrm{VI}$ \\
\end{tabular} & ARI & MI & $k$ & VI & ARI & MI & $k$ & VI & ARI & MI \\
\hline \multirow{3}{*}{ PGPnet } & Louvain & 44.022 & 0.004 & 0.124 & 30 & 3.94 & $\mathbf{0 . 0 7 3}$ & 1.102 & & 04.08 & 0.041 & 0.87 \\
\hline & Infomap & 43.583 & 0.006 & 0.1 & 30 & 3.7 & 0.1 & 0.98 & & 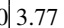 & 0.063 & 0.78 \\
\hline & Bigclam & $4 \quad 1.57$ & 0.011 & 0.029 & 30 & 3.4 & 0.12 & 1.026 & & 03.462 & 0.07 & 0.78 \\
\hline \multirow{3}{*}{ Cond-mat } & Louvain & 34.021 & 0.01 & 0.225 & 52 & 3.91 & 0.011 & 0.324 & 52 & \begin{tabular}{|l|l|}
2 & 4.5 \\
\end{tabular} & 0.04 & 0.68 \\
\hline & Infomap & 33.132 & 0.05 & 0.222 & 52 & 3.1 & 0.051 & 0.32 & & 23.63 & 0.224 & 0.68 \\
\hline & Bigclam & \begin{tabular}{|l|l|}
3 & 0.5 \\
\end{tabular} & 0.49 & 0.562 & 52 & 1.47 & 0.155 & 2.49 & 52 & 21.22 & 0.331 & 1.99 \\
\hline \multirow{3}{*}{$\mathrm{HepPh}$} & Louvain & \begin{tabular}{|l|l|}
3 & 3.22 \\
\end{tabular} & 0.028 & 0.093 & 76 & 5.7395 & 0.014 & 0.216 & & 65.23 & 0.09 & 0.64 \\
\hline & Infor & 35.72 & 0.006 & 0.166 & 76 & 7.224 & 0.014 & 0.78 & 76 & 66.3 & 0.1 & 1.4 \\
\hline & Bigclam & 30.95 & 0.0004 & 0.0005 & 76 & 6.44 & 0.001 & 0.51 & & $6 \quad 6.25$ & 0.09 & 0.8 \\
\hline \multirow{3}{*}{ Enron } & Louvain & \begin{tabular}{|l|l|}
3 & 3.6 \\
\end{tabular} & 0.038 & 0.274 & 16 & 3.57 & $\mathbf{0 . 0 5 3}$ & 0.442 & 16 & 63.51 & 0.0441 & 0.37 \\
\hline & Inf & 1.6 & 0.467 & 0.261 & 16 & 1.73 & 0.53 & 0.4 & 16 & 61.62 & 0.51 & 0.344 \\
\hline & Bigclam & 0.7 & 0.48 & 0.51 & 16 & 0.91 & 0.51 & 1.97 & 16 & 60.94 & 0.50 & 1.3 \\
\hline \multirow{3}{*}{ Epinion } & Louvain & 3.1 & 0.0006 & 0.005 & 24 & \begin{tabular}{|l|}
3.08 \\
\end{tabular} & 0.001 & 0.015 & & 43.451 & 0.03 & 0.06 \\
\hline & Infon & 34.163 & 0.017 & 0.007 & 24 & 4.16 & 0.031 & 0.013 & 24 & 44.47 & 0.014 & 0.1 \\
\hline & Bigclam & \begin{tabular}{|l|l|}
3 & 0.11 \\
\end{tabular} & 0.1 & 0.007 & 24 & 0.84 & 0.02 & 0.02 & 24 & $\begin{array}{ll}4 & 0.74\end{array}$ & 0.014 & 0.016 \\
\hline \multirow{3}{*}{ Imdb-actor } & Louvain & \begin{tabular}{|l|l|}
3 & 3.05 \\
\end{tabular} & 0.0005 & 0.006 & 59 & 4.4 & 0.114 & 1.09 & & \begin{tabular}{l|l|}
9 & 3.84
\end{tabular} & 0.093 & 0.673 \\
\hline & Infomap & \begin{tabular}{|l|l|}
3 & 2.7 \\
\end{tabular} & 0.0006 & 0.004 & 59 & 4.2 & 0.11 & 0.99 & & $\begin{array}{l}9 \\
9.58\end{array}$ & 0.08 & 0.63 \\
\hline & Bigclam & 1.1 & 0.0 & 0.0 & 59. & 3.0405 & 0.025 & 1.07 & 59 & $\begin{array}{l}9 \\
9\end{array}$ & 0.02 & 0.83 \\
\hline \multirow{3}{*}{ Youtube } & Louvain & \begin{tabular}{|l|l|}
3 & 3.64 \\
\end{tabular} & 0.0003 & 0.004 & 21 & \begin{tabular}{|l|}
4.1 \\
\end{tabular} & 0.016 & 0.047 & 21 & 14.55 & 0.017 & 0.096 \\
\hline & Infomap & \begin{tabular}{ll|}
3 & 3.71 \\
\end{tabular} & 0.0003 & 0.0035 & 21 & 4.16 & 0.014 & 0.03 & 21 & \begin{tabular}{l|l|}
1 & 4.7
\end{tabular} & 0.016 & 0.07 \\
\hline & Bigclam & 30.056 & 0.0 & $2.6 \mathrm{e}-06$ & 21 & 1.6 & 0.035 & 0.06 & & 11.25 & 0.09 & 0.1 \\
\hline \multirow{3}{*}{ roadCA } & Louvain & \begin{tabular}{|l|l|}
3 & 6.12 \\
\end{tabular} & 0.0004 & \begin{tabular}{|l|}
0.141 \\
\end{tabular} & 39 & 6.38 & 0.0005 & 0.41 & & $\begin{array}{ll}9 & 6.42\end{array}$ & 0.0006 & 0.242 \\
\hline & Infomap & \begin{tabular}{|l|l|}
3 & 8.0 \\
\end{tabular} & $7.6 e-05$ & 0.21 & 39 & 7.9 & 0.0001 & 0.64 & & 98.14 & 0.00011 & 0.40206 \\
\hline & Bigclam & \begin{tabular}{|lll} 
& 1.075 \\
\end{tabular} & 0.0 & 0.0 & 39 & 2.62 & 0.044 & 1.26 & 39 & $\begin{array}{ll}9 & 1.56\end{array}$ & 0.17 & 1.01 \\
\hline
\end{tabular}

TABLE II: Evaluation of the cluster memberships for Original KSC, ST-KSC and $k$-means KSC for several real world networks w.r.t. Louvain, Infomap and Bigclam methods. The highlighted numbers represent the best results. Original KSC method performs better w.r.t. VI while the ST-KSC and ST $k$-means KSC methods perform well w.r.t. ARI and MI.

Bigclam method.

From Figure $6 \mathrm{~b}$ we observe that the clusters obtained by all the KSC methods have high VI and low ARI values in comparison to the clusters obtained by Louvain and Infomap method. This is because the mean number of clusters obtained by Louvain and Infomap method are 3,000 and 65,807 respectively over 10 randomizations. This is much larger in comparison to the number of clusters obtained by Original KSC (3) and 39 obtained by the proposed approach. However, the Bigclam method identifies 40 clusters. The clusters obtained by Bigclam method have best result w.r.t. VI with Original KSC method (1.075), w.r.t. ARI with ST $k$-means KSC (0.17) and w.r.t. MI with ST-KSC (1.26).

\section{CONCLUSION}

In this paper we proposed an approach to make the kernel spectral clustering method free of parameters for large scale networks. We used the normalized linear kernel for large scale networks and devised an approach to automatically identify the number of clusters $k$ in the given network. For achieving this, we exploit the projections of the validation nodes in the eigenspace by creating an affinity matrix which had block-diagonal structure. We used the concepts of entropy and balance to identify these block-diagonals and obtain the number of clusters. We compared the resulting KSC methods with large scale community detection methods like Louvain, Infomap and Bigclam methods. In future work, we plan to evaluate the clusters obtained by the KSC methods using other likelihood based techniques like BIC and AIC.

\section{Acknowledgements}

This work was supported by Research Council KUL, ERC AdG A-DATADRIVE-B, GOA/10/09MaNet, CoE EF/05/006, FWO G.0588.09, G.0377.12, SBO POM, IUAP P6/04 DYSCO, COST intelliCIS.

\section{REFERENCES}

[1] Danaon, L., Diáz-Guilera, A., Duch, J., Arenas, A. Comparing community structure identification. Journal of Statistical Mechanics: Theory and Experiment, 09(P09008+), 2005.

[2] Fortunato, S. Community detection in graphs. Physics Reports, 2009, 486, 75-174.

[3] Clauset, A., Newman, M., Moore, C. Finding community structure in very large scale networks. Physical Review E, 2004, 70(066111).

[4] Girvan, M., Newman, M. Community structure in social and biological networks. PNAS, 2002, 99(12), 7821-7826.

[5] Lancichinetti, A., Fortunato, S. Community detection algorithms: a comparitive analysis. Physical Review E, 2009, 80(056117).

[6] Rosvall, M. and Bergstrom, C. Maps of random walks on complex networks reveal community structure. PNAS, 2008, 105, 1118-1123.

[7] Langone, R., Alzate, C., Suykens, J.A.K. Kernel spectral clustering for community detection in complex networks. In IEEE WCCI/IJCNN, 2012, 2596-2603.

[8] Blondel, V., Guillaume, J., Lambiotte, R., Lefebvre, L. Fast unfolding of communities in large networks. Journal of Statistical Mechanics: Theory and Experiment, 2008, 10(P10008).

[9] Ng, A.Y., Jordan, M.I., Weiss, Y. On spectral clustering: analysis and an algorithm, In proceedings of the Advances in Neural Information Processing Systems; Dietterich, T.G., Becker, S., Ghahramani, Z., editors, MIT Press: Cambridge, MA, 2002; pp. 849-856.

[10] von Luxburg, U. A tutorial on Spectral clustering. Stat. Comput,2007, $17,395-416$.

[11] Zelnik-Manor, L., Perona, P. Self-tuning spectral clustering. Advances in Neural Information Processing Systems; Saul, L.K., Weiss, Y., Bottou, L., editors; MIT Press: Cambridge, MA, 2005; pp. 1601-1608.

[12] Shi, J., Malik, J. Normalized cuts and image segmentation. IEEE TPAMI, 2000, 22(8), 888-905.

[13] Schaeffer, S. Algorithms for Nonuniform Networks. Phd thesis, Helsinki University of Technology, 2006.

[14] Alzate, C, Suykens, J.A.K. Multiway spectral clustering with out-ofsample extensions through weighted kernel PCA. IEEE Transactions on Pattern Analysis and Machine Intelligence, 2010, 32(2), 335-347.

[15] Mall, R., Langone, R., Suykens, J.A.K. Kernel Spectral Clustering for Big Data Networks, Entropy (Special Issue: Big Data), 2013, 15(5), 1567-1586.

[16] Newman, M.E.J. Modularity and community structure in networks. PNAS, 2006, 103(23), 8577-8582.

[17] Maiya, A., Berger-Wolf, T. Sampling community structure. $W W W, \mathbf{2 0 1 0}$, 701-710.

[18] Mall, R., Langone, R., Suykens, J.A.K. FURS: Fast and Unique Representative Subset selection for large scale community structure, Internal Report 13-22, ESAT-SISTA, K.U.Leuven, 2013.

[19] Kang, U., Faloutsos, C. Beyond 'caveman communities': Hubs and Spokes for graph compression and mining. In Proceedings of ICDM, 2011, 300-309.

[20] Metropolis, N., Rosenbluth, A., Rosenbluth, M., Teller, A., Teller, E. Equation of state calculations by fast computing machines. Journal of Chem. Phys., 1953, 21(6), 1087-1092.

[21] Leskovec, J., Faloutsos, C. Sampling from large graphs. KDD, 2006.

[22] Langone, R., Alzate, C., Suykens, J.A.K. Kernel Spectral Clustering with Memory Effect. Physica A: Statistical Mechanics and its Applications, 2012, 392(10), 2588-2606.

[23] Chung, F.R.K.; Spectral Graph Theory. American Mathematical Society, 1997.

[24] Suykens, J.A.K., Van Gestel, T., De Brabanter, J., De Moor, B., Vandewalle, J. Least Squares Support Vector Machines. World Scientific, Singapore, 2002.

[25] Muflikhah, L. Document Clustering using Concept Space and Cosine Similarity Measurement. ICCTD, 2009, 58-62.

[26] Baylis, J. Error Correcting Codes: A Mathematical Introduction. 1988.

[27] Hubert, L., Arabie, P. Comparing partitions. Journal of Classification, 1985, 2, 193-218.

[28] Yang, J., Leskovec, J. Overlapping community detection at scale: a nonnegative matrix factorization approach. In Proceedings of WSDM, 2013, 587-596.

[29] Rabbany, R., Takaffoli, M., Fagnan, J., Zaiane, O.R., Campello R.J.G.B. Relative Validity Criteria for Community Mining Algorithms. 2012, International Conference on Advances in Social Networks Analysis and Mining (ASONAM), 258-265. 